\title{
Increase in spleen volume as a predictor of oxaliplatin toxicity
}

This article was published in the following Dove Press journal:

Therapeutics and Clinical Risk Management

\author{
Alissar El Chediak' \\ Ali A Haydar ${ }^{2}$ \\ Ayman Hakim' \\ Sarah Abdel Massih' \\ Lara Hilal ${ }^{3}$ \\ Deborah Mukherji' \\ Sally Temraz' \\ Ali Shamseddine' \\ 'Department of Internal Medicine, \\ Division of Hematology-Oncology, \\ American University of Beirut-Medical \\ Center, Beirut, Lebanon; ${ }^{2}$ Department \\ of Diagnostic Radiology, American \\ University of Beirut-Medical Center, \\ Beirut, Lebanon; ${ }^{3}$ Department of \\ Radiation Oncology, American \\ University of Beirut-Medical Center, \\ Beirut, Lebanon
}

Background: Oxaliplatin is a nonconventional third-generation platinum compound. It is an important chemotherapeutic agent in regimens used in gastrointestinal carcinomas as well as other malignancies. Oxaliplatin toxicity profile includes neurotoxicity, hepatotoxicity, and splenomegaly. The primary aim of this study was to measure the spleen volume of patients on oxaliplatin therapy before and during chemotherapy to detect any increase in splenic size as a biomarker for early oxaliplatin toxicity.

Methods: This was a prospective pilot study conducted at the American University of BeirutMedical Center. Fifty patients newly started on oxaliplatin were included. The spleen volume was measured from the patients' baseline CT scan using the IntelliSpace Portal upgraded system (using Response Evaluation Criteria In Solid Tumors [RECIST]), for each follow-up CT scan. Side effects were evaluated at each patient visit and graded according to the severity.

Results: Thirty-seven (74\%) patients developed an increase in spleen size. Thirty-three (66\%) sampled patients developed peripheral neuropathy (all grades) at 3 months, whereas only two (4\%) patients developed grade 3 neuropathy. Only one (3\%) patient who developed an increase in spleen size also developed grade 3 peripheral neuropathy - a result that is significantly different $(p<0.001)$ when comparing patients with an increase in spleen size who also developed peripheral neuropathy of other grades.

Conclusion: An increase in spleen volume possibly precedes a significant peripheral neuropathy which could be a potential marker for oxaliplatin-induced toxicity.

Keywords: oxaliplatin, toxicity, neuropathy, splenomegaly, neuropathy, hepatic sinusoidal injury

\section{Introduction}

Oxaliplatin is a nonconventional third-generation platinum compound, which differs from cisplatin in its lack of nephrotoxicity and from carboplatin in its association with less hematological toxicity and bone marrow suppression. ${ }^{1}$ The clinical development of oxaliplatin-based regimens started in the 1990s. Oxaliplatin-based therapy is currently being used in colorectal cancer in the adjuvant, neoadjuvant, particularly for resectable or initially unresectable liver metastasis, and the metastatic settings. ${ }^{2,3}$ FOLFOX, an oxaliplatin fluorouracil (5-FU)/folinic acid (FA) regimen, consistently improves the response rates and progression-free survival in metastatic colorectal cancer. ${ }^{4}$ Alternatively, capecitabine with oxaliplatin $\left(\mathrm{Xeloda}^{\circledR}\right)$ is a feasible combination regimen that shows the promising antitumor activity in patients with colorectal cancer. ${ }^{5}$

Oxaliplatin also represents an important chemotherapeutic agent in regimens used in other gastrointestinal carcinomas such as esophageal cancer or pancreatic cancer as well as other malignancies such as ovarian, breast, or even non-Hodgkin's lymphoma. ${ }^{6,7}$ 
Oxaliplatin's most frequent and clinically relevant adverse event is neurotoxicity. It mainly manifests as peripheral neuropathy that is cumulative and dose limiting. Nausea, vomiting, stomatitis, and mucositis are also reported. Hepatotoxicity is another adverse event manifested in the increase in transaminases and alkaline phosphatase ${ }^{6}$ secondary to hepatic sinusoidal injury. This was first reported by Rubbia-Brandt et $a l,{ }^{8}$ who showed that the development of severe hepatic sinusoidal injury in resected liver specimens strongly correlates with oxaliplatin use; 34 out of 43 patients (78\%) treated with oxaliplatin showed striking sinusoidal alterations. These alterations have many similarities to the changes seen in the sinusoidal obstruction syndrome, which can theoretically lead to signs and symptoms of portal hypertension. ${ }^{2}$ Clinical and pathological analyses following oxaliplatin-based neoadjuvant chemotherapy regimens showed an association between hepatic sinusoidal injury, portal hypertension, splenomegaly, and thrombocytopenia. ${ }^{2}$ Several studies highlighted that the degree of the increase in splenic size strongly correlates with the reduction in platelets count; ${ }^{2,6}$ thus, splenic sequestration is one of the proposed mechanisms of oxaliplatin-induced thrombocytopenia. ${ }^{7}$ As for more serious fatal side effects, overt liver failure and death have been rarely reported., ${ }^{2,9}$

The development of hepatic sinusoidal injury after oxaliplatin treatment has not been directly correlated with mortality increase but rather manifested as an increase in postsurgical morbidity, bleeding risk, the need for blood transfusions, and hospitalization period. Thus, a method for predicting the severity of hepatic sinusoidal injury would be highly useful for patients receiving oxaliplatin, specifically for those who are planned for possible surgeries.

Increase in splenic size has served as a biomarker for the risk of oxaliplatin-induced hepatic sinusoidal injury. ${ }^{2}$ However, the measurement of the splenic size, more specifically splenic volume, can be tricky. Evaluation of splenic size by palpation can be extremely inaccurate given that the spleen is not palpable before it enlarges two to three times its normal size. ${ }^{10}$ Various imaging modalities, including conventional radiography, nuclear scans, ultrasonography, CT scans, and magnetic resonance (MR) scans, have been used to study the spleen. ${ }^{11}$ Volumetric measurements whether based on the predetermined formulae or the commercially available volumetric software are most accurately obtained on CT or MR imaging. ${ }^{12-14}$

The primary aim of this study was to measure the splenic volume of patients on oxaliplatin therapy, before and during chemotherapy, to detect any increase in splenic size as a surrogate for early oxaliplatin toxicity. Other clinical indicators of oxaliplatin toxicity such as peripheral neuropathy or an increase in transaminases were also measured and correlated with possible advent increase in splenic size.

\section{Methods}

\section{Study design and patient recruitment}

This is a prospective pilot study that was conducted at the American University of Beirut-Medical Center (AUB-MC), a large tertiary referral center in Lebanon. The study protocol was approved by the Institutional Review Board of the American University of Beirut. Fifty patients having different types of cancers who newly started on oxaliplatin were recruited from Oncology private clinics and inpatient wards at AUB-MC, and written informed consent was obtained from all the patients. Private clinic medical records were reviewed in order to complete the medical information.

Patients have received several regimens of oxaliplatin in the following forms and dosages:

Neoadjuvant setting: ${ }^{15}$

- mFOLFOX: Oxaliplatin $85 \mathrm{mg} / \mathrm{m}^{2}$ intravenous (IV) over $2 \mathrm{~h}$; leucovorin $400 \mathrm{mg} / \mathrm{m}^{2} \mathrm{IV}$ over $2 \mathrm{~h}$; 5 -FU $400 \mathrm{mg} / \mathrm{m}^{2}$ IV bolus on day 1 , then $1,200 \mathrm{mg} / \mathrm{m}^{2} /$ day for 2 days continuous infusion. This regimen was repeated every 2 weeks.

- FOLFIRINOX: Oxaliplatin $85 \mathrm{mg} / \mathrm{m}^{2} \mathrm{IV}$ over $2 \mathrm{~h}$; irinotecan $180 \mathrm{mg} / \mathrm{m}^{2}$ IV over 30-90 min; leucovorin $400 \mathrm{mg} / \mathrm{m}^{2}$ IV infusion to match duration of irinotecan infusion on day $1 ; 5-F U 400 \mathrm{mg} / \mathrm{m}^{2} \mathrm{IV}$ bolus on day 1 , then $1,200 \mathrm{mg} / \mathrm{m}^{2} /$ day for 2 days continuous infusion. This regimen was repeated every 2 weeks.

- XELOX: Oxaliplatin $130 \mathrm{mg} / \mathrm{m}^{2} \mathrm{IV}$ over $2 \mathrm{~h}$; capecitabine $1,000 \mathrm{mg} / \mathrm{m}^{2}$ orally twice daily for 14 days. This regimen was repeated every 3 weeks.

Adjuvant setting: ${ }^{15}$

- mFOLFOX6: Oxaliplatin $85 \mathrm{mg} / \mathrm{m}^{2} \mathrm{IV}$ over $2 \mathrm{~h}$; leucovorin $400 \mathrm{mg} / \mathrm{m}^{2} \mathrm{IV}$ over $2 \mathrm{~h} ; 5$-FU $400 \mathrm{mg} / \mathrm{m}^{2}$ IV bolus on day 1 , then $1,200 \mathrm{mg} / \mathrm{m}^{2} /$ day for 2 days (total $2,400 \mathrm{mg} / \mathrm{m}^{2}$ over 46-48 h) continuous infusion. This regimen was repeated every 2 weeks.

- FOLFIRINOX: Oxaliplatin $85 \mathrm{mg} / \mathrm{m}^{2} \mathrm{IV}$ over $2 \mathrm{~h}$; leucovorin $400 \mathrm{mg} / \mathrm{m}^{2}$ IV over $2 \mathrm{~h}$; irinotecan $180 \mathrm{mg} / \mathrm{m}^{2}$ IV over $90 \mathrm{~min}$, concurrently with leucovorin; 5-FU $400 \mathrm{mg} / \mathrm{m}^{2} \mathrm{IV}$ bolus, after leucovorin then $400 \mathrm{mg} / \mathrm{m}^{2}$ IV continuous infusion over $46 \mathrm{~h}$.

- XELOX: Oxaliplatin $130 \mathrm{mg} / \mathrm{m}^{2} \mathrm{IV}$ over $2 \mathrm{~h}$; capecitabine $1,000 \mathrm{mg} / \mathrm{m}^{2}$ orally twice daily on days $1-14$, every 3 weeks for eight cycles. 
Initial spleen volume was measured from the patients' baseline CT scan using the IntelliSpace Portal (ISP) upgraded system that draws a 3D schematic representation of the spleen to calculate the splenic volume. Volumetric measurements were repeated upon several follow-up CTs. Also, side effects related to oxaliplatin were evaluated at each patient visit and graded according to the Common Terminology Criteria for Adverse Events (CTCAE) (grades I, II, and III).

Patients with preexisting liver cirrhosis, prior splenectomy, or absence of baseline spleen imaging at AUB-MC were excluded. The percentage change in spleen size compared to baseline, ie, prior to initiation of chemotherapy, is reported, rather than comparison to a certain cutoff value of spleen volume. Mean difference with SD in splenic size progression during chemotherapy course is also reported.

\section{Statistical analysis}

All statistical analyses were performed using SPSS software. The splenic volume before and during chemotherapy will be compared using the paired $t$-test. A difference is considered significant when the $p$-value is $\leq 0.05$. Correlational studies between the increase in spleen size and other known oxaliplatin side effects were done using chi-square and paired $t$-tests.

\section{Results}

Patient characteristics are shown in Table 1. The median age at diagnosis was 60 years.

Table I Characteristics of the patients

\begin{tabular}{|c|c|c|c|c|}
\hline Parameters & Value & $\begin{array}{l}\text { Percentage } \\
(\mathrm{N}=50)\end{array}$ & Total & Missing \\
\hline \multirow[t]{2}{*}{ Gender } & Male & $74(n=37)$ & 50 & 0 \\
\hline & Female & $26(n=13)$ & & \\
\hline Type of & Colon & $50(n=25)$ & 50 & 0 \\
\hline \multirow[t]{3}{*}{ cancer } & Pancreatic & $22(n=I I)$ & & \\
\hline & Rectal & $16(n=8)$ & & \\
\hline & Gastric & $12(n=6)$ & & \\
\hline \multirow[t]{3}{*}{ Stage } & IV & $58(n=29)$ & 50 & 0 \\
\hline & III & $34(n=17)$ & & \\
\hline & II & $8(n=4)$ & & \\
\hline Neoadjuvant & FOLFOX & $16(n=8)$ & 15 & 0 \\
\hline \multirow[t]{2}{*}{ chemotherapy } & FOLFIRINOX & $12(n=6)$ & & \\
\hline & XELOX & $2(n=1)$ & & \\
\hline Adjuvant & FOLFOX & $40(n=20)$ & 35 & 0 \\
\hline \multirow[t]{2}{*}{ chemotherapy } & FOLFIRINOX & $16(n=8)$ & & \\
\hline & XELOX & $14(n=7)$ & & \\
\hline \multirow[t]{4}{*}{ Metastasis } & None & $42(n=2 I)$ & 50 & 0 \\
\hline & Liver & $48(n=24)$ & & \\
\hline & Lung & $2(n=1)$ & & \\
\hline & Others & $8(n=4)$ & & \\
\hline
\end{tabular}

Table 2 Adverse events of oxaliplatin

\begin{tabular}{llll}
\hline Events & Yes, \% & No, \% & Missing \\
\hline Increase in spleen size & $74(n=37)$ & $24(n=12)$ & I \\
Thrombocytopenia & I0 $(n=5)$ & $88(n=44)$ & I \\
Peripheral neuropathy & GI: $42(n=21)$ & $34(n=17)$ & 0 \\
& G2: $20(n=10)$ & & \\
G3: $4(n=2)$ & & \\
AST increase & I4 (n=7) & $44(n=22)$ & 21 \\
ALT increase & I4 (n=7) & $46(n=23)$ & 20 \\
\hline
\end{tabular}

Abbreviations: ALT, alanine transaminase; AST, aspartate transaminase.

Median increase in spleen volume at 3 months was $31 \%$ with a maximum increase of $119 \%$.

Thirty-seven (74\%) patients developed an increase in spleen size. Thirty-three (66\%) sampled patients developed peripheral neuropathy (all grades) at 3 months, of which only two (4\%) patients developed grade 3 neuropathy (Table 2).

Using crosstabs analysis, we examined the relationship between the percentage increase in spleen size and the following variables: thrombocytopenia $(p=0.8)$, leucopenia ( $p=0.05)$, peripheral neuropathy ( $p=0.7)$, and increase in liver transaminases $(p=0.07)$. Therefore, the increase in spleen size was not correlated with any of those variables.

Subgroup analysis has shown that $11 \%(p=1)$ of those who developed an increase in spleen size also developed thrombocytopenia and $49 \%$ ( $p=0.8)$ of those who developed an increase in spleen size also developed peripheral neuropathy. One (3\%) patient who developed an increase in spleen size also developed grade 3 peripheral neuropathy. This value turned out to be statistically significant $(p<0.001)$ when comparing patients with an increase in spleen size who also developed peripheral neuropathy of other grades.

\section{Discussion}

Oxaliplatin constitutes an integral medication in the armamentarium against several types of cancers, mainly colorectal cancer. Nonetheless, it is associated with toxicities. Particularly, hepatotoxicity is a concern to treating physicians. It is associated with an increase in liver amylases. This is thought to be due to liver sinusoidal injury, which presents with histopathological changes similar to the changes seen in the sinusoidal obstruction syndrome..$^{16-18}$

Most of the imaging modalities used to assess the spleen have certain disadvantages in providing accurate volume estimations of the spleen. Radiography and scintigraphy assume a regular geometric shape of the spleen and only linear measurements are performed; however, the spleen is characterized by its irregular shape, which varies widely 
among individuals. Moreover, measurements of spleen volume using sonography are often limited by the incomplete scanning of the spleen or by the incomplete visualization of the organ due to the presence of overlying structures (such as the left kidney, bowel gas, or bone). ${ }^{11,19}$

An increase in spleen size also occurs with oxaliplatin use. ${ }^{20}$ Thirty-seven (74\%) of our patients developed an increase in spleen size (Table 2). In a study of Korean patients, 50 participants receiving oxaliplatin were evaluated to detect changes in spleen size and platelet counts. ${ }^{21}$ The incidence of splenomegaly (67\%) after 12 cycles of oxaliplatin was close to that found in our study. ${ }^{21}$

Another frequent side effect of oxaliplatin use is thrombocytopenia. Clinical trials reported it in $40 \%$ of the patients. $^{22}$ Nonetheless, the incidence of thrombocytopenia in our sample was $10 \%$ (Table 2). Some studies reported a relation between splenomegaly, portal hypertension, and thrombocytopenia. ${ }^{2,20}$ So, a possible explanation for the low incidence of thrombocytopenia in our sample can be because liver injury has not occurred yet but might develop in future cycles affecting the platelet count.

Since the development of hepatic sinusoidal injury leads to portal hypertension and results in splenomegaly, increase in spleen size has been suggested as a marker of oxaliplatininduced hepatotoxicity. ${ }^{20,21}$ In a study with 79 patients who received oxaliplatin-based therapy, Imai et $\mathrm{al}^{3}$ identified the increase in the splenic volume as an independent predictor of the development of sinusoidal obstruction syndrome. A study done on 136 patients in MD Anderson Cancer Center treated with adjuvant oxaliplatin and 5-FU showed that the increase in spleen volume is an independent predictor of the development of sinusoidal obstruction syndrome. ${ }^{2}$ Moreover, Park et $\mathrm{al}^{23}$ evaluated the correlation between chemotherapyassociated changes of noninvasive liver fibrosis indices and volumetric changes of the spleen. They found that the changes in noninvasive liver fibrosis indices showed a good correlation with the increase in the spleen volume during oxaliplatin-based chemotherapy. All these support the use of the increase in spleen volume as an indicator of oxaliplatin hepatotoxicity.

Only one patient developed an increase in spleen size along with grade 3 peripheral neuropathy, a result that is significantly different when comparing the development of the increase in spleen size with peripheral neuropathy of lower grades. Thus, it can be hypothesized that developing an increase in spleen size possibly precedes a significant peripheral neuropathy (grade 3 ), allowing this increase to be a potential marker for oxaliplatin-induced toxicity. Possible mechanisms of neurotoxicity include decreased digital nerve conduction velocities with neuronal atrophy as suggested by Renn et al. ${ }^{24}$ Oxaliplatin also causes an increase in the activity of neurons in the spinal dorsal horn resulting in allodynia and cold hyperalgesia. ${ }^{24,25}$ All these mechanisms require confirmatory testing with nerve studies. This further demonstrates the role of increase in spleen size as a predictor for oxaliplatin neurotoxicity as well as liver toxicity. The importance of this finding is emphasized by the need for noninvasive predictors of oxaliplatin toxicity. A liver biopsy is needed to diagnose sinusoidal obstruction syndrome, a mechanism for oxaliplatin-induced liver injury. By contrast, noninvasive predictors of sinusoidal obstruction syndrome such as the increase in spleen volume have a strong advantage in terms of providing prognostic information of clinical relevance and with regard to patient safety and cost. ${ }^{26}$ Further studies with a larger sample and histological comparison are needed to validate these findings.

\section{Conclusion}

Oxaliplatin-induced liver injury presents an increase in morbidity especially for patients undergoing resection of metastasis to the liver in gastrointestinal tumors. Noninvasive methods are needed to predict oxaliplatin toxicity. An increase in spleen volume might be an indicator of the development of oxaliplatin-induced toxicity before clinical manifestation of the toxicity, which needs to be further explored in larger studies.

\section{Acknowledgments}

The abstract of this paper was presented at the ESMO 19th World Congress on Gastrointestinal Cancer, June 28-July 1, 2017, Barcelona, Spain, and was published in Annals of Oncology, Volume 28, Issue suppl 3 June 2017.

\section{Author contributions}

All authors contributed toward data analysis, drafting and revising the paper and agree to be accountable for all aspects of the work

\section{Disclosure}

The authors report no conflicts of interest in this work.

\section{References}

1. Misset JL. Oxaliplatin in practice. Br J Cancer. 1998;77(Suppl 4): 4-7.

2. Overman MJ, Maru DM, Charnsangavej C, et al. Oxaliplatin-mediated increase in spleen size as a biomarker for the development of hepatic sinusoidal injury. J Clin Oncol. 2010;28(15):2549-2555.

3. Imai K, Emi Y, Iyama KI, et al. Splenic volume may be a useful indicator of the protective effect of bevacizumab against oxaliplatininduced hepatic sinusoidal obstruction syndrome. Eur J Surg Oncol. 2014;40(5):559-566. 
4. Simpson D, Dunn C, Curran M, Goa KL. Oxaliplatin: a review of its use in combination therapy for advanced metastatic colorectal cancer. Drugs. 2003;63(19):2127-2156.

5. Diaz-Rubio E, Evans TR, Tabemero J, et al. Capecitabine (Xeloda) in combination with oxaliplatin: a phase I, dose-escalation study in patients with advanced or metastatic solid tumors. Ann Oncol. 2002; 13(4):558-565.

6. Hoff PM, Saad ED, Costa F, et al. Literature review and practical aspects on the management of oxaliplatin-associated toxicity. Clin Colorectal Cancer. 2012;11(2):93-100.

7. Jardim DL, Rodrigues CA, Novis YA, Rocha VG, Hoff PM. Oxaliplatinrelated thrombocytopenia. Ann Oncol. 2012;23(8):1937-1942.

8. Rubbia-Brandt L, Audard V, Sartoretti P, et al. Severe hepatic sinusoidal obstruction associated with oxaliplatin-based chemotherapy in patients with metastatic colorectal cancer. Ann Oncol. 2004;15(3):460-466.

9. Tisman G, MacDonald D, Shindell N, et al. Oxaliplatin toxicity masquerading as recurrent colon cancer. J Clin Oncol. 2004;22(15): 3202-3204.

10. De Odorico I, Spaulding KA, Pretorius DH, Lev-Toaff AS, Bailey TB, Nelson TR. Normal splenic volumes estimated using three-dimensional ultrasonography. J Ultrasound Med. 1999;18(3):231-236.

11. Asghar A, Agrawal D, Yunus SM, Sharma PK, Zaidi SH, Sinha A Standard splenic volume estimation in North Indian adult population using 3D reconstruction of abdominal CT scan images. Anat Res Int 2011;2011:707325.

12. Musa AF, Abdo DAM, Zidan MMA, Ali WM, Khojaly SN. Estimation of splenic volume in Sudanese adult population using abdominal computed tomography scan. Int J Med Imaging. 2017;5(1):1-4.

13. Prassopoulos P, Daskalogiannaki M, Raissaki M, Hatjidakis A, Gourtsoyiannis N. Determination of normal splenic volume on computed tomography in relation to age, gender and body habitus. Eur Radiol. 1997;7(2):246-248.

14. Harris A, Kamishima T, Hao HY, et al. Splenic volume measurements on computed tomography utilizing automatically contouring software and its relationship with age, gender, and anthropometric parameters. Eur J Radiol. 2010;75(1):e97-e101.

15. Benson AB, 3rd, Venook AP, Cederquist L, Chan E, Chen YJ, Cooper HS, et al. Colon Cancer, Version 1, 2017, NCCN Clinical Practice Guidelines in Oncology. Journal of the National Comprehensive Cancer Network: JNCCN. 2017;15(3):370-398.
16. Mehta NN, Ravikumar R, Coldham CA, et al. Effect of preoperative chemotherapy on liver resection for colorectal liver metastases. Eur $J$ Surg Oncol. 2008;34(7):782-786.

17. Nakano H, Oussoultzoglou E, Rosso E, et al. Sinusoidal injury increases morbidity after major hepatectomy in patients with colorectal liver metastases receiving preoperative chemotherapy. Ann Surg. 2008; 247(1):118-124

18. Vauthey JN, Pawlik TM, Ribero D, et al. Chemotherapy regimen predicts steatohepatitis and an increase in 90-day mortality after surgery for hepatic colorectal metastases. J Clin Oncol. 2006;24(13): 2065-2072.

19. Mazonakis M, Damilakis J, Maris T, Prassopoulos P, Gourtsoyiannis N. Estimation of spleen volume using MR imaging and a random marking technique. Eur Radiol. 2000;10(12):1899-1903.

20. Angitapalli R, Litwin AM, Kumar PR, et al. Adjuvant FOLFOX chemotherapy and splenomegaly in patients with stages II-III colorectal cancer. Oncology. 2009;76(5):363-368.

21. Jung EJ, Ryu CG, Kim G, et al. Splenomegaly during oxaliplatin-based chemotherapy for colorectal carcinoma. Anticancer Res. 2012;32(8): $3357-3362$

22. sanofi-aventis. ELOXATIN ${ }^{\circledR}$ [prescribing information]. Bridgewater Township, NJ; 2015:14

23. Park S, Kim HY, Kim H, et al. Changes in noninvasive liver fibrosis indices and spleen size during chemotherapy: potential markers for oxaliplatin-induced sinusoidal obstruction syndrome. Medicine. 2016; 95(2):e2454

24. Renn CL, Carozzi VA, Rhee P, Gallop D, Dorsey SG, Cavaletti G. Multimodal assessment of painful peripheral neuropathy induced by chronic oxaliplatin-based chemotherapy in mice. Mol Pain. 2011;7:29.

25. Kokotis P, Schmelz M, Kostouros E, Karandreas N, Dimopoulos MA. Oxaliplatin-induced neuropathy: a long-term clinical and neurophysiologic follow-up study. Clin Colorectal Cancer. 2016;15(3): e133-e140.

26. Patel K, Bedossa P, Castera L. Diagnosis of liver fibrosis: present and future. Semin Liver Dis. 2015;35(2):166-183.
Therapeutics and Clinical Risk Management

\section{Publish your work in this journal}

Therapeutics and Clinical Risk Management is an international, peerreviewed journal of clinical therapeutics and risk management, focusing on concise rapid reporting of clinical studies in all therapeutic areas outcomes, safety, and programs for the effective, safe, and sustained use of medicines. This journal is indexed on PubMed Central, CAS,

\section{Dovepress}

EMBase, Scopus and the Elsevier Bibliographic databases. The manuscript management system is completely online and includes a very quick and fair peer-review system, which is all easy to use. Visit http://www.dovepress.com/testimonials.php to read real quotes from published authors. 